\title{
Application of general anesthesia as part of dental care for children with special needs
}

\begin{abstract}
Ambarkova Vesna* Sv.Pantelejmon Skopje 1000 , Republic of Macedonia

Children with special needs are group of patients suffering from various systemic disorders that requires medical management, health care interventions and specialized services or programs. Oral health is often overlooked in children with special needs [1]. Although the approach to children with disabilities хаве undergone changes over time, dental care for these patients is usually boiled down only to emergency treatment, the tooth extraction, which is still going on in many communities, the less developed and poorer areas. Oral diseases have a great impact on the quality of children's life [2].
\end{abstract}

Faculty of Dental Medicine, Department of Paediatric and Preventive Dentistry, University St. Cyril and Methodius, Vodnjanska 17 University Dental Clinic Center

From the 1997, at the Clinic of Paediatric and Preventive Dentistry within the University Dental Clinic Center Ss.Pantelejmon, the teeth can be restored and treated under general anesthesia. It is primarily introduced for uncooperative or handicapped children and adults who cannot be treated regularly in a dental office. All the necessary interventions can be undertaken during a single visit. Currently, we are the only institution in the country that offers services of this kind on a highly professional level.

The treatment under general anesthesia is conducted according to the most rigorous safety protocols. The operating room for these interventions is supplied with all the necessary equipment, and the anesthesia itself is conducted by a specialist - anesthesiologist.

Induced loss of consciousness by medication during which the patient does not respond even to painful stimuli is a postulate of general anesthesia in which the ability of spontaneous breathing is often violated. It is necessary during general anesthesia to maintain a patent airway of the patients, and is also sometimes necessary to make active ventilation due to depression of neuromuscular function. Cardiovascular function may also be affected. As a deep sedation and general anesthesia have no sharp boundaries, it is not always possible to predict how each patient would react.

Therefore, clinicians who plan to carry out the treatment with the use of a certain level of sedation, they must be able to recognize and respond appropriately to all the physiological consequences caused by the level of sedation, if it turns out that the sedation is deeper than the one that was previously planned. Indications for general anesthesia are: autism, mental retardation, cerebral paralysis, but most indications are closely related to the degree of disability.

Special caution, during giving general anesthesia in patients with a disease from the group of muscular dystrophies is require. Contraindications for general anesthesia are influenced by current general health condition of the patient.

Factors influencing the treatment plan of children undergoing general anesthesia are: identification and differentiation of patients with special needs and assessment of their opportunities for cooperation.
Specifics of dental care under general anesthesia are that the patients cannot keep your mouth open during the dental interventions and the use of special factory-produced props and mouth openers is require. Since the treatments on teeth are performed per quadrant, when mouth props are set on one side, dentist works on teeth from the opposite quadrants of the upper and lower jaws.

There are four types of general anesthesia: intravenous, intramuscular, inhalation and combined.

The intravenous anesthesia is used for short-term interventions or as a introduction of the handicapped child in inhalation anesthesia. The most common inhaled anesthetics are barbiturates, ketamine drugs, etomidate drugs, neuroleptics and benzodiazepines.

Inhaled commonly used anesthetics are ether, chloroform, halothane, nitrous oxide, cyclopropane. They are used for short-term intervention or in the stage of introduction in general anesthesia.

Mixed (balanced) anesthesia involves the use of several medications that enter in the body of the patient through the various entries in order to achieve general anesthesia. Anesthesiologist performed emphasizing the desirable effects of medication until the unwanted effects are reduced to a minimum. There is no particular pattern after which applies to all combined anesthesia, it is planned and implemented individually.

\section{Evaluation of the patient}

Planning of dental care under general anesthesia include:

1. Preservation of the existing health

2. Plan of preventive and prophylactic measures

3. Diagnosis and treatment of dental caries

4. Diagnosis and treatment of complications of caries

5. Radical dental care

6. Regular check-ups

Correspondence to: Vesna Ambarkova, PhD.MSc, DDS, Faculty of Dental Medicine, Department of Paediatric and Preventive Dentistry, University St. Cyril and Methodius, Vodnjanska 17 University Dental Clinic Center Sv.Pantelejmon Skopje 1000, Republic of Macedonia, Tel. ++38970686333, E-mail: vesna.ambarkova@gmail.com

Received: June 02, 2017; Accepted: June 13, 2017; Published: June 15, 2017 
The planning phases of the dental care of children in general anesthesia:

PHASE I - the first admission, classification and preparation of patients with special needs,

PHASE II - determining plan for dental care need.

Patients who are planning to undergo treatment under deep sedation or general anesthesia must be carefully examined before starting any procedure. In healthy people, this examination must include the current general health condition and information about medications that the patient possibly takes at the moment. Before conducting general anesthesia within the health compromised patients, it is necessary consultation and agreement by the specialist of the primary disease.

Preoperative preparation means that the patient, parent, legal guardian must be informed about all facts connected with the procedure which is planned to be carried out, as well as with the use of sedatives or anesthetics and have to give written consent for the proposed intervention. It is necessary to provide sufficient quantities of oxygen and the necessary equipment for the ventilation of the patient and at the beginning of treatment it is necessary to record all vital parameters, if the patient permits, or if he/she cooperate during these procedures. It is also necessary to conduct a detailed clinical examination of the general health of the patient. In the preoperative period, patient is required to received verbal and written instructions in relation to the intervention and venous access must be established. The patient must be constantly monitored until the complete waking up in the operating room.

Monitoring should include: the patient oxygenation, the color of the skin and mucous membranes must be accompanied at all times, oxygen saturation must be continuously monitored by pulse oximeter, the ventilation, circulation and the temperature.

During the entire intervention, it is necessary the anesthetic nurse to write a proper anesthetic list:

The used drugs

- Doze

- The recorded physiological parameters.

- Recovery and discharges of the patient

- The system of ventilation and aspiration must be currently available;

- The dentist or any member of the team should continuously observe the patient's blood pressure, pulse, saturation and the level of awareness;

- Documentation that the degree of consciousness, breathing and circulation functions are at acceptable levels must be written, before the discharge of the patient

Postoperative verbal and written recommendations must be given to the patient, his or her parents or guardians.

Dental treatment under general anesthesia required to make

- closing of the pharynx with gauze,

- setting mouth opener

- aspiration of the contents of the oral cavity.

The usual sequences of dental treatment are: the removal of plaque, the removal of calculus, restorations of teeth, fissure sealing and at last oral surgery interventions.

Specifics treatment plan include avoiding of dental procedures in many sessions and procedures that require laboratory phases are almost contraindicated. When remediation milk teeth dentition should be restored only cavities that do not threaten the vitality. All suspected deep carious lesions, gangrenous primary teeth and dental radices are removed, do not leave the open gangrenous teeth to keep the space. Priority are given to supporting teeth zone V, III, IV. When dental care is conducting within mixed dentition, absolute priority is given to permanent teeth, and dental care of the primary teeth that remain longest in the mouth, and dental care of proximal caries with a radical approach.

Restorative materials used for front teeth are glass ionomer cements, risen modified glass-ionomer cements and compomers, while for posterior teeth with dental caries of I, II and MOD classes we used glass ionomer cements, risen modified glass-ionomer cements, composites and compomers, which have less than four years of survival. The dental materials for primary molars teeth, which have more than four years of survival are metal crowns and amalgam.

Individual prevention program is essential before applying general anesthesia, this program also means motivation of parents and is carried out in order to prevent the emergence of a new caries lesions, secondary caries and periodontal disease.

Side effects include: pain in the mouth, a headache, nausea and vomiting.

\section{Medical documentation includes}

- Dental data registered in the dental carton for Children and Adolescent

- Anesthesiologist take care for diary during anesthesia

- Discharge list

Keeping records can have multiple (clinical, forensic-medical, administrative-financial and epidemiological) significance.

\section{Controversies in connection with the application of general anesthesia}

- the use of force, oral, intravenous, inhalation sedation or general anesthesia must be viewed as different clinical forms of coercion.

- general anesthesia, with all of its benefits, must be regarded as superior clinical technique of coercion.

- falling asleep patient who lacks capacity to consent to treatment even take away the ability to deduct

\section{Treatment}

- sedation also result in the patient's reduced ability to reject the treatment.

The advantages of the Clinic of Pediatric and Preventive dentistry are:

- A team consisting of top professionals in this field;

- Technical equipment (most up-to-date apparatuses and instruments); 
- Application of contemporary materials, manufactured by world renowned producers;

- Application of most current methods and techniques;

- More than 50 years tradition in the field of paediatric dentistry.

The main disadvantage of the Clinic of Pediatric and Preventive dentistry is long time waiting for general anesthesia. Currently 250 patients are on the waiting list for general anesthesia.

The average waiting time in Morocco was 7.8 months [3], while in our clinic is about one year. Only two days in every week, anesthesiologist comes at our department, usually performing two children under general anesthetics per day. In the future, we should work to create better conditions for everyday operation under general anesthesia. This will improve oral health special need children in our country.

\section{References}

1. Chen CY, Chen YW, Tsai TP, Shih WY (2014) Oral health status of children with special health care needs receiving dental treatment under general anesthesia at the dental clinic of Taipei Veterans General Hospital in Taiwan. J Chin Med Assoc 77: 198-202.[Crossref]

2. Baens-FerrerC, Roseman MM, Dumas HM, Haley SM (2005)Parental perceptions of oral health-related quality of life for children with special needs: Impact of oral rehabilitation under general anesthesia.Pediatr Dent 27: 137-142.[Crossref]

3. Badre B, Serhier Z, El Arabi S (2014) Waiting times before dental care under general anesthesia in children with special needs in the Children's Hospital of Casablanca. Pan Afr Med J 17: 298. [Crossref]

Copyright: (C2017 Vesna A. This is an open-access article distributed under the terms of the Creative Commons Attribution License, which permits unrestricted use, distribution, and reproduction in any medium, provided the original author and source are credited. 